\title{
A comprehensive approach to quality management of intensive care services
}

Seetharaman Hariharan and Prasanta Kumar Dey

\section{Introduction}

Recently, there has been an increased need for intensive care units (ICU) owing to aggressive therapeutic interventions for critically ill patients (Barbieri et al., 2003). Critical care is one of healthcare's high-technology and high-budget areas, and most countries strive hard to cope with increasing demand for ICU facilities (Hariharan et al., 2007). On one hand, ICU availability may be the primary issue (especially in the developing world); while on the other, unit performance is a wider global issue. Optimal healthcare process performance is essential if services are to remain competitive. Today's healthcare providers deploy quality management tools and techniques to improve customer satisfaction, although not routinely (Lurie et al., 2002). Data-Attitude-Tools, Deming's "plan, do, check and act" (PDCA) cycle, Utilization Review and Management have been applied to healthcare services (Van Matre, 1992; Feeney and Zairi, 1996; Berwick, 1998). Continuous quality improvement (CQI) has been applied to many healthcare areas most notably in Emergency Departments (Re and Krousel-Wood, 1990; Fernandes and Christenson, 1995, Fernandes et al., 1996).

Applying quality management tools and techniques to improve performance is relatively new to intensive care services. Peer-review process, a common method to identify ICU deficiencies (Stow et al., 2006), is usually done at mortality/morbidity meetings, case conferences and reviewing patient data. Another performance evaluation method is applying various prognostic models (Becker and Zimmerman, 1996). Clinicians may use physiological scoring systems for assessing illness severity and relate them to outcomes. In fact, models such as the Acute Physiology and Chronic Health Evaluation (APACHE) and the Simplified Acute Physiology Score (SAPS) have been continuously evolving to improve their ability to assess ICU performance (Moreno et al., 2005; Zimmerman et al., 2006). Despite their refinement, by applying stricter statistical criteria, most prognostic models are plagued with the so-called "goodness-of-fit" issues. This implies that models may not be able to predict outcomes when applied to a different case-mix other than the one used to design them (Sherck and Shatney, 1996).

Using prognostic models, clinicians determine the unit's expected mortality and then compare it with observed mortality. If the observed mortality is higher than expected for instance, it may only point out generally that there may be unit deficiencies. This approach does not identify ICU specific problems and does not provide a framework for adopting strategies needed for correction and improvement. Unit managers may have to design their own framework from performance appraisal to the strategy implementation. Hence there is a need for a model that provides all quality improvement aspects as an all-inclusive package for the manager. Our earlier research applied analytic hierarchy process (AHP) for ICU performance measurement through benchmarking input, processes and output (Hariharan et al., 2005). Although the proposed framework identifies issues needing to be addressed, it does not suggest a project management framework to plan, implement and evaluate improvement projects. Therefore, there is strong need for a comprehensive quality management approach to improve ICU performance. Our objective, therefore, is to help ICU clinicians identify quality issues, identify improvement projects, plan, implement and evaluate those projects in order to improve performance.

\section{Method}

Our study adopts an interactive approach involving stakeholders (clinicians, hospital managers, patients and their relatives) and group consensus to develop the model and use it to derive improvement measures. We formulated the model using the following steps (also shown in Figure 1):

1. A system is selected for study on the basis of its performance.

2. The causes for not achieving desired performance are identified using a cause and effect diagram.

3. A logical framework (LF) is then developed using cause and effect diagram information. 
4. A detailed project plan is developed.

5. Improvement projects are implemented and evaluated to determine progress.

6. Continuous quality improvement is implemented through repeating the cycle dynamically.

The following paragraphs explain the cause and effect diagram, LF and the combined cause and effect diagram and LF.

\section{The cause and effect diagram}

Cause and effect (also known as fishbone and Ishikawa) diagrams, developed by Ishikawa in 1968, help users to think through problem causes (Ishikawa, 1986). The approach combines brainstorming and a concept map. The process has four major steps: identifying the problem; working out the major factors involved; identifying possible causes; and analyzing the cause and effect diagram, which are used to resolve numerous problems including risk management in production and services (Dey, 2004). The technique has been well applied to obstetrics and gynecology, and emergency department healthcare management (White et al., 2004, 2005).

The $L F$

A LF is an analytical management tool that helps to:

- analyze existing situations during project preparation;

- establish a logical hierarchy by which objectives will be reached;

- identify potential risks to achieving objectives and to sustainable outcomes;

- establish how outputs and outcomes might best be monitored and evaluated;

- present a project summary in a standard format; and

- monitors and reviews projects during implementation.

The approach involves problem analysis, stakeholder analysis, developing hierarchical objectives and selecting a preferred implementation strategy. The product is a matrix (the logframe), which summarizes the project's intentions and modus operandi. Its key assumptions, monitoring methods and evaluating outputs and outcomes are shown in Table I.

In the early 1970s, LFs were formally adopted by the United States Agency for International Development (USAID) as a planning tool for overseas development activities. They have been successfully applied as a planning and management tool by many agencies. Despite criticisms, it has been proven popular and its use continues to expand into new areas because it helps to provide a standardized project summary. LFs have been applied in many healthcare projects, including: implementing national level projects in HIV/AIDS, malaria, etc. (AusGUIDE, 2005; World Health Organization, 1999; Department of Health, Zambia, 2000). However, to our knowledge, a LF has not been applied to ICUs for quality improvement purposes.

\section{The combined cause and effect approach and LF}

The cause and effect diagram helps identify issue root causes (quality problem) while the logframe helps to plan and evaluate project dynamically to control project time, cost and quality. This combination concurs with the Deming's total quality management PDCA cycle. The method was applied to the Barbados Queen Elizabeth Hospital surgical ICU, part of a 600-bed tertiary care teaching hospital affiliated to the University of the West Indies. The ICU is a sixbed open unit admitting patients from general wards, operating rooms and emergency department.

\section{Method}

Identifying reasons for not achieving desired performance using a cause and effect diagram Initially, questionnaire surveys were conducted among all the unit's stakeholders including 30 staff and 30 patient/relatives. The staff included clinical professionals in the ICU such as medical, nursing and paramedical personnel. The survey's main objective was to determine the unit's major issues likely to dissatisfy staff. Stakeholder survey data were analyzed and categorized into the main problems considered to be the primary parameters for the 
processes being investigated. Audits conducted earlier and outcomes data were also incorporated to determine the current major issues affecting the ICU's performance. The main problems were grouped into four main categories:

1. infrastructure;

2. human resources;

3. hospital-related units; and

4. ICU organization.

Next, individual factors felt to cause these four major problems were determined - identified using brainstorming sessions involving ten clinicians having more than 15 years' ICU experience. Additionally, data and outcomes from existing audit and research were also included to construct the cause and effect diagram (Figure 2).

Analyzing individual factors revealed the following deficiencies contributing to the problems.

\section{Infrastructure}

Lack of adequate infrastructure and equipment were identified as a major problem. The Post Anaesthesia Care Unit (PACU) was found to serve as an additional ICU, admitting ICU patients almost daily. Increasing ICU beds was the foremost infrastructure priority. Although the ICU could physically admit more patients, improper infrastructure and equipment maintenance were other reasons limiting the ICU.

\section{Personnel}

There was understaffing in all categories causing overworking. Often, instead of the normal 1:1, the nurse to patient ratio was 1:2, respectively, thereby compromising care. Similarly, junior medical officers frequently had to take ICU "on call" (sometimes alternate days), which again increased their burden. This led to doctor, nurse and support staff commitment issues, which also diminished teamwork.

\section{Related units}

There were delayed referrals from wards and the Accident and Emergency (A\&E) Department, which resulted in more severely ill patients admitted to the ICU. The delay caused multiple organ systems dysfunction, thereby increasing patient morbidity and mortality. There were also delays obtaining laboratory and radiology reports owing to networking deficiencies. There were delays ordering drugs and supply was often poor, especially cardiovascular medicines required for day-to-day patient management. Audits revealed that many patients suffered cardiac arrest during transfer from A\&E to the ICU owing to improper patient monitoring.

\section{ICU organization}

Absence of organized daily rounds by senior physicians and junior medical officers "on duty" was identified as the major day-to-day management problem. Other problems were lacking treatment protocols resulting in treatment not based on current evidence-based guidelines. A dearth of admission/discharge protocols resulted in moribund inpatients and increased stays. Nonexistent antibiotic protocols led to a wider choice of antimicrobials, which predisposed higher resistance rates. There were also poor infection control measures in place and the infection control nurse did not visit the ICU weekly.

\section{Mitigation}

Clinicians decided after extensive brainstorming sessions that the following strategies would improve performance:

- Purchasing state-of-the-art equipment, establishing an equipment preventive maintenance program, establishing information technology (IT), drug and equipment invoicing to maintain a supply-chain to improve infrastructure.

- The following measures were suggested to improve staffing: increased full-time equivalents, fair salaries, improvised working conditions especially working hours, promoting and rewarding staff, and team development activities and establishing a trained ICU management team. 
- Information technology to link laboratories and radiology services for prompt access to reports. Regular morbidity/mortality meetings should include related staff to highlight problems associated with delayed referrals and formulating guidelines for intra-hospital transport were suggested.

- Bringing about organizational change, formulating admission/discharge, treatment, antibiotic protocols, ensuring adherence to the above protocols, establishing an organized daily rounds involving senior staff, regular audits and case conferences and scheduled inputs from infection control staff were also suggested as improvement measures.

\section{Developing a LF for planning, implementing and evaluating improvement projects} A LF was developed, which formed the entire improvement project's objective planning parameters (Figure 3). Subsequent planning, design and implementation brought about the desired improvements.

\section{Results}

Information collected using surveys, audits and hospital records regarding all quality improvement parameters were applied into the logframe to determine if there was any difference after the quality improvement exercise. The same parameters were re-measured in 2004 and compared with the 2002 results (before improvement measures were implemented), which revealed performance improvement. Currently, the project is at various levels of implementation in the hospital. More staff have been recruited; salaries and incentives increased, which in turn motivated staff. An ad hoc ICU director was appointed who coordinated many quality improvement measures. Treatment, admission and discharge protocols were formulated. An antibiotic protocol was implemented with the hospital microbiologist's help, baseline infections and resistance patterns in the ICU have been set.

Each quality improvement activity was implemented through detailed planning, designing and procurement. We use a communication improvement activity as an example (Figure 4). The ICU we studied is an open unit and patients are managed by both ICU physicians and the admitting physicians. This activity's main purpose was to improve communication between ICU and interdepartmental staff as well as patients and surrogates. Initially, in order to develop the project scope, the cause and effect diagram was used from which the LF (Figure 4) was developed. The LF acted as initial project plan, which helped to develop a detailed plan.

Subsequently, the following processes were adopted to implement effective communication:

- Identify ICU stakeholders.

- Identify each stakeholder's communication requirements.

- Identify current communication means (software, hardware and protocol/process).

- Identify communication gaps using the expectation vs perception survey (Table II).

- Suggest and implement improvements (software, hardware, protocol and training).

- Measure performance after implementation and show improvement.

The following paragraphs demonstrate each step.

\section{Identifying ICU stakeholders}

Consultant intensivists, junior medical officers, nurse-in-charge, ICU nurses and support staff were identified as intradepartmental stakeholders. Parent unit consultants, A\&E physicians, referring specialists, including surgeons, cardiologist, microbiologist, radiologists, physiotherapists and social workers were considered interdepartmental stakeholders. Patients and surrogates were considered external ICU customers.

\section{Identifying each stakeholder's communication requirements}

Establishing intradepartmental communication between physicians, physicians to nurses, and nurses to support staff was the first requirement. Establishing interdepartmental communication involving stakeholders from other departments was the second. Establishing effective communication between ICU personnel and patients as well as relatives and their surrogates was the third. 


\section{Identifying current communication means}

Intra ICU communication was poor as there were no organized consultant intensivist or junior physician dailyrounds. Interdepartmental communication was also not effective because there was no proper record. Additionally, there were no structured sessions between ICU personnel, patient or relatives.

\section{Identifying current gaps in communication by expectation vs perception survey}

A service quality measurement (SERVQUAL) survey (Table II) was conducted to derive ICU stakeholder communication expectations vs. perception. The details and validity of this type of survey in health services has been discussed elsewhere (Babakus and Mangold, 1992). We observed that the average difference between expectation and perception was more than 30 percent.

\section{Suggesting and implementing improvements}

The ICU physicians and nurses developed daily patient management plans through organized daily rounds, which established effective patient care according to evidence-based protocols. The case presentation seminars, which were held weekly, helped to discuss issues related to morbidity and adverse outcomes. Similar sessions involving other departmental staff were held monthly. Thus effective communication about various cases between multispecialty physicians was established. Other staff (e.g. physiotherapists and radiology technicians) maintained both physical and computerized specific patient service records to keep nurses updated. Patient referrals were recorded manually and electronically, which helped to monitor patient care by various specialties. An interdepartmental communication system was established using information technology, which enabled effective patient movement from the A\&E to ICU and from ICU to high dependency unit and general wards. Biochemical and pathological reports were accessible in the ICU by linking laboratory and ICU computers and this enabled faster and better patient management. Patient relatives were updated about the patient's condition each day by the senior registrar during evening visiting hours. The nurse-in-charge was required to be present during these sessions. Consultant physicians also participated when critical decisions on patient-care were made. The hospital chaplain regularly visited the ICU and interacted with patients and their relatives.

\section{Measuring performance on implementation and observing improvement}

Another survey was carried out to determine expected and perceived ICU performance after the "communication improvement" activity was implemented. Results revealed that the post change difference between expected and perceived service performance was 5 percent. This was a considerable achievement. Similar approaches were undertaken for other quality improvement areas and incorporated into the framework.

\section{Discussion}

Healthcare delivery is broadly a continuous service and any model for improving healthcare should not ideally be a "one-time" approach. There should be periodical healthcare service problems reviews from a stakeholder perspective for CQI. Our model offers a new approach to ICU CQI. Quality improvement from concept to implementation is a challenging task. It involves not only fostering a learning culture in the organization but also planning and implementing a framework for each concept. Healthcare quality is usually assessed using three parameters: structure, process and outcome (Donabedian, 1988). Currently, outcome-based models dominate ICU performance measurement and quality improvement. These outcome-based models exclusively consider binomially either "death" or "survival" as a quality indicator; however they may not be useful by themselves as an overall performance indicator (Glance et al., 2002). In fact there is opinion that prognostic models most often evaluate ICUs as highly performing and there could be a grade-inflation effect if units are assessed only according to outcome (Popovich, 2002). Hence, quality improvement measures should include two other parameters: ICU infrastructure and care process. Our earlier research applying an analytic hierarchy process for performance evaluation also found that care process is an important parameter (Hariharan et al., 2005).

A recent study identified the most important ICU "quality indicators" and found that intensivist availabiliyu (hours per day), patient-to-nurse ratio and patient/family satisfaction measurement were four major indicators (de Vos et 
al., 2007). Intensive care patients and the family members' opinion and satisfaction are one of the most important ICU quality improvement aspects (Gallesio et al., 2006). The Society of Critical Care Medicine Task Force in the USA recently provided an overview for quality improvement using a step-wise approach, although no specific framework or model was recommended (Curtis et al., 2006). Additionally, the Task Force also identified four steps for sustaining this program:

1. determine whether the target is changing;

2. modify behavior change strategies to improve or sustain improvements;

3. focus on interdisciplinary collaboration; and

4. develop and sustain support from hospital leaders.

The present framework, with minimal modifications to suit the local perspectives, could enable such programs in any ICU. Several units in a particular region could apply this model uniformly to compare their individual quality improvement measures. Multiple-hospital collaboration is found to be important for improving critical care delivery processes (McMillan and Hyzy, 2007).

Applying a LF model to the ICU is a global approach necessitating appraisal of all three healthcare delivery quality measures. When different ICU management dimensions are taken into perspective, it offers improvement measures in many areas that have been missed using routine peer-reviews and audits. One of the present model's many benefits is that it includes issues that would not have otherwise received adequate attention. Establishing good communication between staff, patient and surrogates is an important quality improvement measure that may not have been prioritized if other quality improvement methods were chosen. Communication was recognized to be deficient and the present approach of addressing this issue specifically dealt with the problem. The exercise enabled us to establish effective communication between staff, patients and surrogates shown by surveys conducted before and after implementing the measures. This approach helped us to formulate short-term and long-term improvement measures. Additionally the model enabled us to follow-up quality improvement activity on a continuous basis.

\section{Conclusion and recommendations}

The LF offers a uniform model that can be applied to most ICUs. It is also possible to incorporate the model into IT, creating an automated application, which facilitates monitoring implementation leading to further quality improvement, thus making this a CQI exercise. Continuous quality improvement requires regular and concurrent performance appraisal. Data collection and analysis can show that improvement measures have been appropriately and effectively implemented (Slovensky, 1996). Although our model could be applied to any ICU, we recommend that it is tailored taking into consideration local and regional factors governing individual ICUs. Additionally, in ICUs where software application and electronic data are available, this facility may enhance the model's application, which was lacking in our situation. We also recommend that surveys for constructing the model and ascertaining its effectiveness should be tailored to suit the ICU's needs, given that stakeholder views may vary according to different healthcare systems. Nevertheless, the combined cause and effect diagram and LF analysis is an effective framework for improving ICU services. It provides an objective framework for planning and evaluating improvement projects. Similar approaches could be adapted to other units to improve performance.

\section{[UNAVAILABLE IN THIS VERSION]:}

Figure 1Management model for ICU quality improvement

Figure 2ICU cause and effect diagram

Figure 3Logical framework for improving ICU performance

Figure 4Logical framework for improving ICU performance

Table ILogical framework structure 


\section{Table IISERVQUAL expectations and perceptions survey}

\section{References}

AusGUIDE (2005), “Completion and evaluation”, available at: www.ausaid.gov.au/ausguide/part5.cfm (accessed September 2008), .

Babakus, E., Mangold, W.G. (1992), "Adapting the SERVQUAL scale to hospital services: an empirical investigation", Health Services Research, Vol. 26 No.6, pp.767-86.

Barbieri, S., Feltracco, P., Michieletto, E., Basso, I., Spagna, A., Giron, G. (2003), "Demand and availability of intensive care beds. A study based on the data collected at the SUEM 118 Central of Padua from October 1996 to December 2001", Minerva Anestesiology, Vol. 69 No.7-8, pp.625-39.

Becker, R.B., Zimmerman, J.E. (1996), "ICU scoring systems allow prediction of patient outcomes or comparison of ICU performance", Critical Care Clinics, Vol. 12 No.3, pp.503-14.

Berwick, D.M. (1998), "Developing and testing changes in the delivery of care", Annals of Internal Medicine, Vol. 128 No.8, pp.651-6.

Curtis, J.R., Cook, D.J., Wall, R.J., Angus, D.C., Bion, J., Kacmarek, R., Kane-Gill, S.L., Kirchhoff, K.T., Levy, M., Mitchell, P.H., Moreno, R., Pronovost, P., Puntillo, K. (2006), "Intensive care unit quality improvement: a how-to guide for the interdisciplinary team", Critical Care Medicine, Vol. 31 No.1, pp.211-8.

de Vos, M., Graafmans, W., Keesman, E., Westert, G., van der Voort, P.H. (2007), "Quality measurement at intensive care units: which indicators should we use?", Journal of Critical Care, Vol. 22 No.4, pp.467-74.

Department of Health, Zambia (2000), "Managing the impact of HIV/AIDS in SADC", available at: www.doh.gov.za/aids/docs/sadc/logical.pdf (accessed September 2008), .

Dey, P.K. (2004), "Decision support system for inspection and maintenance: a case of oil pipelines", IEEE Transactions on Engineering Management, Vol. 51 No.1, pp.47-56.

Donabedian, A. (1988), "The quality of care - how can it be assessed?", Journal of American Medical Association, Vol. 260 No.12, pp.1743-8.

Feeney, A., Zairi, M. (1996), "TQM in healthcare", Journal of General Management, Vol. 22 No.1, pp.35-47.

Fernandes, C.M., Christenson, J.M., Price, A. (1996), "Continuous quality improvement reduces length of stay for fast-track patients in an emergency department", Academic Emergency Medicine, Vol. 3 No.3, pp.258-63.

Fernandes, C.M.B., Christenson, J.M. (1995), "Use of continuous quality improvement to facilitate patient flow through the triage and fasttrack areas of an emergency department", Journal of Emergency Medicine, Vol. 13 No.6, pp.847-55.

Gallesio, A.O., Ceraso, D., Palizas, F. (2006), "Improving quality in the intensive care unit setting", Critical Care Clinics, Vol. 22 No.3, pp.547-71.

Glance, L.G., Osler, T.M., Dick, A. (2002), "Rating the quality of intensive care units: Is it a function of the intensive care scoring system?", Critical Care Medicine, Vol. 30 No.9, pp.1976-82.

Hariharan, S., Chen, D., Merritt-Charles, L. (2007), "Cost evaluation in the intensive care units of Trinidad applying the cost-blocks method - an international comparison", Anaesthesia, Vol. 62 No.3, pp.244-9.

Hariharan, S., Dey, P.K., Chen, D., Moseley, H.S.L., Kumar, A.Y. (2005), "Analytic hierarchy process for measuring and comparing the global performance of intensive care units", Journal of Critical Care, Vol. 20 No.2, pp.17-125.

Inter-American Development Bank (2008), available at: www.iadb.org (accessed January 2008), .

Ishikawa, K. (1986), Guide to Quality Control, 2nd rev. ed., Asian Productivity Organization, Tokyo, .

Lurie, J.D., Merrens, E.J., Lee, J., Splaine, M.E. (2002), "An approach to hospital quality improvement", Medical Clinics of North America, Vol. 86 No.4, pp.825-45.

McMillan, T.R., Hyzy, R.C. (2007), "Bringing quality improvement into the intensive care unit", Critical Care Medicine, Vol. 35 No.2, (Supplement), pp.S59-S65.

Moreno, R.P., Metnitz, P.G., Almeida, E. (2005), "SAPS 3 - from evaluation of the patient to evaluation of the intensive care unit. Part 2: development of a prognostic model for hospital mortality at ICU admission", Intensive Care Medicine, Vol. 31 No.10, pp.1345-55. 
Popovich, M.J. (2002), "If most intensive care units are graduating with honors, is it genuine quality or grade inflation?", Critical Care Medicine, Vol. 30 No.9, pp.2145-6.

Re, R.N., Krousel-Wood, M.A. (1990), "How to use continuous quality improvement theory and statistical quality control tools in a multidisciplinary clinic", Quality Review Bulletin, Vol. 16 No.11, pp.391-7.

Sherck, J.P., Shatney, C.H. (1996), "ICU scoring systems do not allow prediction of patient outcomes or comparison of ICU performance", Critical Care Clinics, Vol. 12 No.3, pp.515-23.

Slovensky, D.J. (1996), "Quality assessment and improvement", in Abdelhak, M., Grostick, S., Hanken, M.A., Jacobs, E. (Eds),Health Information: Management of a Strategic Resource, WB Saunders, Philadelphia, PA, pp.320-57.

Stow, P.J., Hart, G.K., Higlett, T., George, C., Herkes, R., McWilliam, D., Bellomo, R. (2006), "Development and implementation of a high-quality clinical database: the Australian and New Zealand Intensive Care Society Adult Patient Database", Journal of Critical Care, Vol. 21 No.2, pp.13341.

Van Matre, J.G. (1992), "The D*A*T approach to total quality management", Journal of American Health Information Management Association, Vol. 63 No.11, pp.38-40.

White, A.A., Pichert, J.W., Bledsoe, S.H., Irwin, C., Entman, S.S. (2005), "Cause and effect analysis of closed claims in obstetrics and gynecology", Obstetrics and Gynecology, Vol. 105 No.5, pp.1031-8.

White, A.A., Wright, S.W., Blanco, R., Lemonds, B., Sisco, J., Bledsoe, S., Irwin, C., Isenhour, J., Pichert, J.W. (2004), "Cause-and-effect analysis of risk management files to assess patient care in the emergency department", Academic Emergency Medicine, Vol. 11 No.10, pp.1035-41.

World Health Organization (1999), “Roll back malaria”, available at: www.rbm.who.int (accessed September 2008), .

Zimmerman, J.E., Kramer, A.A., McNair, D.S., Malila, F.M. (2006), "Acute Physiology and Chronic Health Evaluation (APACHE) IV: hospital mortality assessment for today's critically ill patients", Critical Care Medicine, Vol. 34 No.5, pp.1297-310. 